\title{
Transient ischemic attacks- Definition, risk prediction and urgent management
}

\author{
P. N. Sylaja, Michael D. Hill ${ }^{1}$
}

Ananthapuri Hospitals and Research Institute, Trivandrum, Kerala, India, Calgary Stroke Program, Department of Clinical Neurosciences, University of Calgary, Calgary, Alberta

\begin{abstract}
Address for correspondence:
Dr. P. N. Sylaja,

Ananthapuri Hospitals and Research

Institute, Trivandrum, India.

E-mail: sylajapn@hotmail.com
\end{abstract}

\begin{abstract}
Recent evidence suggests that the risk of stroke in first few months after transient ischemic attack is higher than that was previously realized. There are clinical and imaging predictors which help in risk stratifying the patients to identify the high risk group who need immediate hospitalization and urgent evaluation. Recent advances in neuroimaging have revolutionized the evaluation of these patients. Further research is required in the deciding on the optimal treatment of these patients in the acute phase.
\end{abstract}

Key words: Neuroimaging, stroke, transient ischemic attacks

\section{Introduction}

Recent data suggest that the burden of transient ischemic attack (TIA) is higher than previously known. About $15-20 \%$ of patients with stroke have a preceding TIA ${ }^{[1]}$ suggesting that these warning events provide us a golden opportunity for stroke prevention. Prospective prognostic studies have clearly shown that the early risk of stroke after TIA is $10-15 \%$ at 90 days. This risk can be reliably estimated by clinical scores, TIA etiology and findings on brain imaging, although a combined prognostic score has not been established. Available evidence suggest that there is a need for urgent evaluation and treatment of these patients to substantially reduce the risk of stroke.

\section{Definition of TIA - Present Controversies}

The traditional definition of TIA is the one published by the Ad Hoc Committee on Cerebrovascular Diseases in 1975 defined as "cerebral dysfunction of ischemic nature lasting no longer than 24 hours with a tendency to recur". ${ }^{[2]}$ Other published definitions include the WHO definition as "sudden focal cerebral dysfunction lasting less than 24 hours of presumed vascular origin confined to the area of brain or eye perfused by a specific artery". We note that clinical experience suggests that many minor strokes are casually called TIA since patients with residual minor symptoms such as mild reduction in finger dexterity or mild paresthesiae in one limb, are most often ignored diagnostically. The use of acute magnetic resonance imaging (MRI) with diffusionweighted (DWI) sequences has shown that many patients with a clinical definition of TIA have small infarcts on imaging. With the recent accumulated data on time duration of TIA and imaging findings, a new tissue based definition has been proposed by Albers and TIA working group and defines TIA as "a brief episode of neurological dysfunction caused by focal brain or retinal ischemia, with clinical symptoms lasting less than an hour and without neuroimaging evidence of acute infarction". ${ }^{[3]}$

\section{Early Stroke Risk after TIA}

Approximately one in 10 patients with TIA experiences a stroke in the next 3 months; this risk may be greater depending upon stroke mechanism. Several studies have evaluated the short term prognosis of TIA. A seminal study of 1707 patients from 16 hospitals in Northern California found a 90 day risk of stroke of $10.5 \%$, and $50 \%$ of this occurred in the first 2 days of TIA. In addition the combined risk of cardiovascular events, death and recurrent TIA was $25.1 \%{ }^{[4]}$ A population based study which examined 2285 patients with TIA diagnosed in Alberta between April 1999 and March 2000 reported a stroke risk of $9.5 \%$ at 90 days and $14.5 \%$ at 1 year. ${ }^{[5]}$ In both of these studies, ascertainment of stroke outcomes was passive, either through chart review or through assessment of administrative data. A systematic review and metaanalysis 
of prospective studies of early risk of stroke after TIA in 10126 TIA patients reported the pooled risk of stroke were $3.1 \%$ [95\%confidence interval (CI)2.0-4.1] at 2 days and $5.2 \%$ (3.9-6.5) at 7 days, with risks ranging from 0 to $12.8 \%$ at 7 days. ${ }^{[6]}$ A metaanalysis of stroke risk after TIA over longer periods of time in 11 studies reported a pooled risk of $8.0 \%(5.7-10.2 \%)$ and $9.2 \%(6.8-11.5 \%)$ at 30 and 90 days respectively ${ }^{[7]}$ Intriguingly, this meta-analysis showed that when TIA was prospectively and actively followed, the risk of recurrence was doubled in the $17.3 \%$ range while when outcomes were sought passively using administrative data, the rate of stroke was approximately $8.7 \%$ at 90 days. ${ }^{[7]}$ A study on the prognosis of stroke and TIA found that the patients with TIA had a greater 6 month recurrence (29\%) than those with stroke $(7 \%){ }^{[8]}$

\section{Risk Prediction after Transient Ischemic Attack}

The risk of stroke after a TIA depends on symptomatology, risk factors and the underlying etiology. In view of the early risk of stroke after TIA, there is a need for urgent evaluation of these patients. But it is not known whether hospitalization of all these patients is cost-effective. Recently risk prediction tools have been developed which can identify the high risk patients in the acute phase after TIA.

\section{Clinical Predictors}

Recently several studies have provided information on the clinical features that identify the patients with TIA at highest risk of stroke. The Northern California study identified age more than 60 years, diabetes mellitus, duration of the episode more than 10 minutes, weakness and speech impairment as factors independently associated with high risk of stroke. Risk varied from $0 \%$ among patients with none of these factors to $34 \%$ among those with all 5. ${ }^{[8]}$ Rothwell et al. reported a six point scoring system - the ABCD rule (age [ $\geq 60$ years $=1]$, blood pressure [systolic $>140 \mathrm{~mm} \mathrm{Hg}$ and /or diastolic $\geq 90 \mathrm{~mm} \mathrm{Hg}=1$ ], clinical features [unilateral weakness $=2$, speech disturbance without weakness $=1$, and other $=0]$, and duration of symptoms in minutes[ $\geq 60=2$, $10-59=1,<10=0$ ] - which was predictive of a 7 day risk of stroke. The early estimated risk of stroke were $0.4 \%$ with a score less than $5,12 \%$ with a score of 5 and $31 \%$ with a score of $6 .{ }^{[9]}$ The ABCD score was refined and revalidated with addition of one point for diabetes to make the ABCD2 score. This $\mathrm{ABCD} 2$ score was highly predictive of the 2 day risk of stroke. ${ }^{[10]}$ In a systematic review and metaanalysis, 11 studies were identified which reported the predictive power of these $A B C D$ scores and found them effective in identifying the true TIA patients at highest risk of stroke. ${ }^{[11]}$
Some caution is warranted in interpreting the ABCD2 clinical prediction rule in routine practice. Firstly, this rule has been well-validated in an isolated British population in Oxford. In Calgary, for example, we found that age $>60$ was sensitive, but highly non-specific and a cut-off of age $>80$ preserved sensitivity and vastly improved specificity [unpublished data]. Secondly, the tool is not designed as a screening test. Most patients who ultimately suffer stroke will have mid-range ABCD2 scores of 2-4 since this is where most patients will fall on the scale. Few patients will fall at the extremes of the scale. Clinically, then the greatest pitfall will lie with the moderate score patient. We have found that the clinical characteristics, motor weakness, visual disturbance and speech/language disturbance are the most important predictors of risk. Nevertheless, the score has provided an important framework for evaluation of TIA patients in the emergency room and added an important focus on the relevant symptoms.

There is a small subset of patients with presumed TIA who have a benign prognosis and develop recurrent TIA and not stroke. History of multiple TIAs, duration of spell less than 10 minutes and isolated sensory symptoms alone have been found to follow a benign course ${ }^{[12]}$ Further, the pattern of recovery from stroke is also important. Patients with rapid early improvement after the onset of neurologic symptoms are at higher risk of subsequent neurological deterioration, indicative of an unstable vasculature. ${ }^{[13,14]}$

\section{Etiology}

The underlying cause of the TIA has been shown to be the major factor that predicts the stroke risk after TIA. A meta analysis of data from 1709 patients showed that the risk of recurrent stroke were $4 \%$ and $12.6 \%$ at 7 days and 30 days respectively in large artery atherosclerotic aetiology compared to $0 \%$ and $2 \%$ in patients with lacunar stroke. ${ }^{[15]}$ Similarly in the study by Eliasziw et al., large artery atherosclerosis has been found to be associated with a high risk of stroke after a TIA. ${ }^{[16]}$ No studies have looked specifically at TIA associated with atrial fibrillation and recurrence rate, perhaps because such patients are most often anticoagulated straight away, preventing any assessment of the natural history.

\section{Territory}

Posterior circulation events constitute about $25 \%$ of TIAs. Previously, these events were considered to have a better prognosis compared to anterior circulation events, but recent evidence suggests that the opposite is true. A meta-analysis, of studies that recruited patients in the acute phase after a TIA event, showed a higher 
risk of stroke after posterior circulation TIA (OR 1.47, 95\% CI 1.1-2.0). ${ }^{[17]}$ Most likely, recurrence depends upon the underlying mechanism of disease. Patients with atherosclerotic vertebrobasilar disease (large artery disease) with plaque rupture and an arteroembolic TIA will be at the highest risk. This mechanism is entirely analogous to the high risk seen with carotid artery plaque rupture at the bifurcation of the common carotid artery.

\section{Neuroimaging Predictors}

Brain imaging has been shown to provide very useful prognostic information, inaddition to confirming the diagnosis and giving information on the territory and etiology of TIA. The presence of infarction on computed tomography (CT) brain scanning in patients with TIA has been shown to be associated with an increased risk of stroke. ${ }^{[18]}$ Though the sensitivity of detecting acute infarction on CT brain is low, its presence is associated with a high risk of stroke recurrence and reduced survival. ${ }^{[1,20]}$ In the study of 322 patients with TIA, new infarct was seen only in $4 \%$, but the risk of stroke during follow-up was substantially higher among those with a new infarct on the head CT(OR,4.06;95\% CI.1.16-14.14; $P=0.028) .{ }^{[20]}$

Diffusion-weighted MRI has been found to be of greater usefulness than CT in patients with TIA. About 40-60\% of patients with TIA have evidence of ischemic injury on DWI suggesting that the clinically transient events are really not transient at the tissue level. ${ }^{[21,22]}$ There are certain factors which decides on the DWI positivity. Symptom duration more than one hour, motor deficits, and aphasia are independently correlated with presence of a DWI lesion. ${ }^{[23]}$ Patients with a DWI lesion were at higher risk of having a subsequent stroke than patients without a lesion. ${ }^{[24,25]}$ The highest risk group were those patients with a DWI lesion and an intracranial occlusion. The 90 day clinical outcome is also closely correlated with DWI lesion and occlusion. Only 2\% of DWI negative/ no intracranial occlusion patients were dependent while $26.7 \%$ with both findings were dependent at 90 days. ${ }^{[25]}$ MRI also has the capability of acting as a more sensitive measure of subclinical ischemic events. In a prospective study of minor stroke and TIA patients $9.8 \%$ revealed new MRI lesions at 30 days follow-up, half of which were clinically silent. The number of DWI lesions at baseline predicted likelihood of new lesions at 30 days. ${ }^{[26]}$ Another study with 360 TIA patients found a higher risk of future stroke in those patients with multiple lesions on DWI, especially if those lesions were of varying ages. ${ }^{[35]}$

\section{Diagnosis of TIA}

The diagnosis of TIA is always clinical. By and large, the diagnosis will also be historical since patients will have fully resolved by the time they are reviewed in the emergency room or in the clinic. However, the clinical diagnosis of TIA is not always straightforward since even focal neurological symptoms can have nonischemic etiologies. Seizure, migraine, syncope, subdural hematoma, intoxication, brain tumour and hypoglycemia may present as a TIA syndrome. ${ }^{[27-30]}$ Patients are variable witnesses to the events that befall them meaning that historical information and details of events are not always reliable. The nature of acute brain dysfunction, for example with hemispatial neglect syndromes, is that some patients will be unaware of their deficits or the severity of illness. A left homonymous hemianopia is rarely recognized acutely due to the associated hemispatial visual neglect. Patients use various words to characterize their symptoms. While "numbness" to a physician implies sensory disturbance, to a patient it may mean weakness or incoordination. Dysarthria and aphasia may be impossible to differentiate historically.

In addition, there is a significant variation among physicians and neurologists in the clinical diagnosis of TIA. In one series, majority ( $81 \%$ ) of the TIA referrals from general practitioners to neurology clinics were for nonvascular events. ${ }^{[31]}$ Even the percent agreement among two neurologists for the diagnosis of TIA by history varies from $42 \%$ to $86 \% .{ }^{[32,33]}$ MRI is helpful because of the high specificity of DWI. However, due to its lower sensitivity, even a normal DWI image should not dissuade the careful neurologist that a good history of TIA is wrong.

\section{Evaluation of Patients with TIA}

A thorough history is essential with emphasis on the onset, symptoms, progression and recovery. CT head scan is important for identifying past stroke and excluding other non-ischemic causes. Imaging the carotids is one of the most important part of the TIA evaluation. This can be done non-invasively using ultrasound, CT angiography (CTA) or MR angiography. CTA compares well with the gold standard digital subtraction angiography. CTA protocols now can include the circle of Willis with the same contrast bolus, providing information regarding the intracranial vasculature and requiring only an additional few minutes of scan time. Combining the results of CT angiography and Doppler ultrasound appears to improve the diagnostic accuracy of identifying carotid stenosis. ${ }^{[34]}$ A 12 lead ECG is mandatory in diagnosing atrial fibrillation. Because the presence of paroxysmal atrial fibrillation will change management, we routinely do 24 hour Holter monitoring on our patients. Echocardiography is restricted to those patients where there is known past cardiac disease or a strong suspicion (eg. based upon MRI) of a cardioembolic source. In practice, for anterior circulation TIA, once 
the carotid arteries have been cleared, both the Holter monitoring and echocardiogram can be done as an outpatient.

Increasingly, it is clear that MRI is the best modality of brain imaging for TIA - its prognostic value is critical for clinical decision making. MR imaging, particularly diffusion weighted imaging is highly sensitive to small volume ischemia. Technical limitations such as slice thickness in the data acquisition can be overcome so that the resolution is in the range of $2-3 \mathrm{~mm}$ in lesion diameter. A further advantage of MR imaging is that the extra-cranial and intra-cranial circulation can be imaged at the same sitting. MR clearly does not provide the same level of resolution of the distal intracranial vasculature compared to $\mathrm{CT}$ angiography but this may not be relevant to immediate management in most cases.

\section{Treatment}

Our practice is to treat TIA as a medical urgency requiring same day assessment. Stratification into high and low risk status can be done relatively quickly in the emergency room [Table 1]. High risk candidates are admitted for urgent evaluation and treatment and low risk patients triaged to clinic. Hospitalization of the high risk TIAs have potential benefits which include: 1) expedited diagnostic evaluation; 2) monitoring of fluctuating patients with ready access to thrombolysis if they deteriorate; ${ }^{[36,37]} 3$ ) facilitation of early carotid revascularization; ${ }^{[38]} 4$ ) greater opportunity for risk factor modification. For effective secondary stroke prevention, current evidence suggest that patients with high risk TIA require rapid referral and a 24 hour admission. ${ }^{[39]}$

The benefit of secondary prevention after TIA has been well established and several treatments have been shown to prevent stroke in the long term including antiplatelet agents ${ }^{[40-43]}$, blood pressure lowering drugs ${ }^{[44]}$,

\begin{tabular}{lll}
\hline \multicolumn{3}{l}{ Table 1: Clinical and imaging } \\
\multicolumn{3}{l}{ Symptodictors of high risk TIA } \\
\hline Clinical predictors & Low risk & High risk \\
Age & $<60$ years & $>60$ years \\
History of diabetes mellitus & No & Yes \\
Motor symptoms & No & Yes \\
Speech symptoms & No & Yes \\
Sensory symptoms only & Yes & No \\
Timing of event & Weeks ago & Hours ago \\
Duration of symptoms & $<10$ minutes & $>60$ minutes \\
Blood pressure & $<140 / 90$ & $>140 / 90$ \\
Frequency of events & Many $(>10)$ & One or few \\
Imaging predictors & & \\
Infarct on CT scan & No & Yes \\
DWI negative & Yes & No \\
Multiple DWI lesions & No & Yes \\
Carotid artery stenosis & No & Yes \\
\hline DWI-diffusion-weighted imaging & & \\
& &
\end{tabular}

statins $^{[45]}$ and carotid endarterectomy ${ }^{[38-46]}$ Till recently we had very little data on benefits of acute treatment after TIA. Two recent studies have shown that above combined prevention treatment started urgently in specialist units can reduce the stroke risk. In the Early use of Existing PREventive Strategies for Stroke (EXPRESS ) study showed that urgent assessment and initiation of a combination of prevention treatments in specialist service can reduce the early recurrent stroke by about $80 \%$ after TIA or minor stroke. ${ }^{[47]}$ A similar good prognosis was seen after urgent and intensive treatment in the SOS-TIA study. ${ }^{[48]}$

\section{Future Directions}

While not emergent, TIA must be treated as a medical urgency that ideally requires same day neurological assessment and investigation. Most gains in the management of TIA will be achieved with logistics management so that patients can be triaged, diagnosed and treated in a timely fashion - we must close the evidence-to-practice gap and actually do what has been proven to work. Therapeutic strategies need to be tested to sort out the best immediate management for these patients. The FASTER-2 trial will test whether the addition of clopidogrel to ASA reduces the early risk of stroke and some 5000 patients will be enrolled. Studies comparing rapid (ie. within $24 \mathrm{~h}$ ) carotid endarterectomy/stenting vs. delayed (ie. within 2 weeks) carotid endarterectomy/stenting are required. Blood pressure management in the acute phase after TIA is entirely uncertain. Many questions remain; the acute management of TIA will be an important subject for randomized trials for some years to come.

\section{References}

1. Rothwell PM, Warlow CP. Timing of transient ischemic attacks preceding ischemic stroke. Neurology 2005;64:817-20.

2. A classification and outline of cerebrovascular diseases: A report by an adhoc committee established by the Advisory council for the National Institute of Neurological Diseases and Blindness, Public Health Service. Neurology 1958;8395-434.

3. Albers GW, Caplan LR, Easton JD, Fayad PB, Mohr JP, Saver JL et al. Transient ischemic attack - proposal for a new definition. N Engl J Med 2002;347:1713-16.

4. Johnston SC, Gress DR, Browner WS, Sidney S. Short-term prognosis after emergency department diagnosis of TIA. JAMA 2000;284:2901-6.

5. Hill MD, Yiannakoulias N, Jeerakathil T, Svenson LW, Schopflocher DP. The high risk of stroke immediately after transient ischemic attack. Neurology 2004;62:2015-20.

6. Giles MF, Rothwell PM. Risk of stroke early after transient ischemic attack : A systematic review and metaanalysis. Lancet Neurol 2007;6:1063-72.

7. Wu CM, McLaughin K, Lorenzetti DL, Hill MD, Manns BJ, Ghali WA. Early risk of stroke after transient ischemic attack : A systematic review and metaanalysis. Arch Intern Med 2007;167:2417-22.

8. Humphrey PR, Marshall J. Transient ischemic attacks and strokes with recovery prognosis and investigation. Stroke 1981;12:765-9.

9. Rothwell PM, Giles MF, Flossman E, Lovelock CE, Redgrave NE, 
Warlow CP et al. A simple score (ABCD) to identify individuals at high early risk of stroke after transient ischemic attack. Lancet 2005; $366: 29-36$.

10. Johnston CS, Rothwell PM, Nguyen-Huynh MN, Giles MF, Elkins JS, Bernstein AL, et al. Validation and refinement of scores to predict very early stroke risk after transient ischemic attack. Lancet 2007;369: 283-92.

11. Giles MF, Rothwell PM. Systematic review and metaanalysis of validations of the ABCD and ABCD2 scores in prediction of stroke risk after transient ischemic attack. Cerebrovasc Dis 2008;25:(Suppl 2):59.

12. Johnston SC, Sidney S, Bernstein AL, Gress DR. A comparison of risk factors for recurrent TIA and stroke in patients diagnosed with TIA. Neurology 2003;60:280-5.

13. Johnston SC, Leira EC, Hansen MD, Adam HP. Early recovery after cerebral ischemia risk of subsequent neurological deterioration. Ann Neurol 2003;54:439-44.

14. Johnston SC, Easton JD. Are patients with acutely recovered cerebral ischemia more unstable? Stroke 2003;34:2446-50.

15. Lovett JK, Coull A, Rothwell PM, on behalf of the Oxford Vascular Study. Early risk of recurrent stroke by aetiological subtype: Implications for stroke prevention. Neurology 2004;62:569-74.

16. Eliasziw M, Kennedy J, Hill MD, Buchan AM, Barnett HJ. Early recovery after a transient ischemic attack in patients with internal carotid disease. CMAJ 2004;170:1105-9.

17. Flossman E, Rothwell PM. Prognosis of vertebrobasilar TIA and minor ischemic stroke. Brain 2003;126:1940-54.

18. van Sweiten JC, Kappelle LJ, Algra, van Latum JC, Koudstaal PJ, van Gijn J. for the Dutch TIA Study Group. Hypodensity of cerebral white matter in patients with transient ischemic attack or minor stroke: Influence on the rate of subsequent stroke. Ann Neurol 1992;32:177-83.

19. Evans GW, Howard G, Murros KE, Rose LA, Toole JF. Cerebral infarction verified by cranial computed tomography and prognosis for survival following transient ischemic attack. Stroke 1991;22:431-36.

20. Douglas VC, Johnston CM, Elkins J, Sidney S, Gress DR, Johnston SC. Head computed tomography findings predict short term risk after transient ischemic attack. Stroke 2003;34:2894-99

21. Ay H, Oliveira-Filho J, Buonanno FS, Schaefer PW, Furie KL, Chang YC et al." Foot prints" of transient ischemic attacks: A diffusionweighted MRI study. Cerebrovasc Dis 2002;14:177-86.

22. Kidwell CS, Alger JR, Di Salle F, Starkman S, Villablanca P, Bentson J et al. Diffusion MRI in patients with transient ischemic attacks. Stroke 1999;30:1174-80.

23. Crisostomo RA, Garcia MM, Tong DC. Detection of diffusion-weighted MRI abnormalities in patients with transient ischemic attack: Correlation with clinical characteristics. Stroke 2003;34:932-7.

24. Purroy F, Montaner J, Rovira A, Delgado P, Quintana M, Alvarez -Sabin J. Higher Risk of Further Vascular Events Among Transient Ischemic Attack Patients With Diffusion-Weighted Imaging Acute Ischemic Lesions. Stroke 2004

25. Coutts SB, Simon JE, Eliasziw M, Sohn CH, Hill MD, Barber PA et al. "Triaging transient ischemic attack and minor stroke patients using acute magnetic resonance imaging." Ann Neurol 2005,57:848-54.

26. Coutts SB, Hill MD, Simon JE, Sohn CH, Scott JN, Demchuk AM. Silent ischemia in minor stroke and TLA patients identified on MR imaging. Neurology 2005,65:513-17.

27. Ay H, Buonanno FS, Rordorf G, Schaefer PW, Schamm LH, Wu O et al. Normal diffusion-weighted MRI during stroke-like deficits. Neurology 1999;52:1784-92.

28. Nor AM, Davis J, Sen B, Shipsey D, Louw SJ, Dyker AG et al. The recognition of stroke in the emergency room (ROSIER) scale: Development and validation of a stroke recognition instrument. Lancet Neurol 2005;4:727-34

29. Libman RB, Wirkowski E, Alvir J, Rao TH. Conditions that mimic stroke in the emergency department. Implications for acute stroke trials. Arch Neurol 1995;52:1119-22.

30. Garcia-Monco JC, Marrodan A, Foncea Beti N, Rao TH, Gomez Beldairain M. Stroke and transient ischemic attack-mimicking conditions: A prospective analysis of risk factors and clinical profiles at a general hospital. Neurologia 2002;17:355-60.

31. Ferro JM, Falcao I, Rodrigues G, Canhao P, Melo TP, Oliveira V et al. Diagnosis of transient ischemic attack by the nonneurologist. A validation study. Stroke 1996;27:2225-9.

32. Tomasello F, Mariani F, Fieschi C, Argentino C, Bono G, De Zanche L et al. Assessment of Interobserver differences in the Italian multicenter study on reversible cerebral ischemia. Stroke 1982;13:32-5.

33. Kraaijeveld CI, Van Gijn J, Schouten HJA, Staal A. Interobserver agreement for the diagnosis of transient ischemic attacks. Stroke 1984; 15:723-5.

34. Patel SG, Collie DA, Wardlaw JM, Lewis SC, Wright AR, Gibson RJ et al. Outcome, observer reliability, and patient preferences if CTA, MRA, or Doppler ultrasound were used individually or together, instead of digital substraction angiography before carotid endarterectomy. J Neurol Neurosurg Psychiatry 2002;73:21-8.

35. Sylaja PN, Coutts SB, Subramanium S, Hill MD, Eliasziw M, Demchuk AM. Acute ischemic lesions of varying ages predict risk of ischemic events in stroke/TIA patients. Neurology 2007;68:415-19.

36. Hill MD, Gladstone DJ. Patients with transient ischemic attack or minor stroke should be admitted to hospital. Stroke 2006;37:1137-8.

37. Nguyen-Huynh MN, Johnston SC. Is hospitalization after TIA costeffective on the basis of treatment with tPA? Neurology 2005;65:1799801

38. Rothwell PM, Eliasziw M, Gutnikov SA, Warlow CP, Barnett HJ. Endarterectomy for symptomatic carotid stenosis in relation to clinical subgroups and timing of surgery. Lancet 2004;363:915-24.

39. Donnan GA, Davis SM, Hill MD, Gladstone DJ. Patients with transient ischemic attack or minor stroke should be admitted to Hospital. Stroke 2006;37:1137-8.

40. Antithrombotic trialists collaboration. Collaborative meta-analysis of randomized trials of antiplatelet therapy for prevention of death, myocardial infarction and stroke in high risk patients. BMJ 2002;324:71-86

41. Kennedy J, Hill MD, Ryckborst KJ, Eliasziw M, Demchuk AM, Buchan AM. For the FASTER investigators. Fast assessment of stroke and transient ischemic attack to prevent early recurrence (FAS'TER): A randomized controlled pilot trial. Lancet Neurol 2007;6:961-69.

42. CAPRIE Steering Committee. A randomized, blinded trial of clopidogrel versus aspirin inpatients at risk of ischemic events (CAPRIE). Lancet 1996;348:1329-39

43. Halkes PH, van Gijn J, Kappelle L.J, Koudstaal PJ, Algra A. ESPIRIT Study Group. Aspirin plus dipyridamole versus aspirin alone after cerebral ischemia of arterial origin (ESPIRIT): Randomized controlled trial. Lancet 2006;367:1665-73.

44. PROGRESS Collaborative Group. Randomized trial of a perindopril based blood-pressure-lowering regimen in 6105 individuals with previous stroke or transient ischemic attack. Lancet 2001;358:1033-41.

45. Amarenco P, Bogousslavsky J, Callahan A III et al. for the Stroke Prevention by Aggressive Reduction in Cholestrol Levels (SPARCL) Investigators. High-does atorvastatin after stroke or transient ischemic attack. N Engl J Med 2006;355:549-559.

46. Rothwell PM, Eliasziv M, Gutnikov SA, Fox AJ, Taylor DW, Mayberg MR, et al. Analysis of pooled data from the randomized controlled trials of endarterectomy for symptomatic carotid stenosis. Lancet 2003;361:107-16.

47. Rothwell PM, Giles MF, Chandratheva A, Marquardt L, Geraghty O, Redgrave JN et al. on behalf of the Early use of Existing Preventive Strategies for Stroke (EXPRESS ) Study. Major reduction in risk of early recurrent stroke by urgent treatment of TLA and minor stroke: EXPRESS Study. Lancet 2007;370:1432-42

48. Lavallee PC, Meseguer E, Abboud H, Cabrejo L, Olivot JM, Simon O et al. A transient ischemic attack clinic with round-the clock access (SOS-TIA): Feasibility and effects. Lancet Neurol 2007;6:953-60.

Accepted on 04-06-09

Source of Support: Nil, Conflict of Interest: None declared. 